\title{
Contribuição para o conhecimento de LAMIINAE (COLEOPTERA: CERAMBYCIDAE) no estado de Mato Grosso, Brasil
}

\author{
Marliton Rocha BARRETO ${ }^{*}$, Pablo João da SILVA ${ }^{1}$ \\ ${ }^{1}$ Instituto de Ciências Naturais, Humanas e Sociais, Universidade Federal de Mato Grosso, Sinop, MT, Brasil. \\ *E-mail: mrb.ufmt@gmail.com
}

Recebido em novembro/2018; Aceito em março/2019.

\begin{abstract}
RESUMO: A Classe Insecta representa $80 \%$ do Reino Animal e a ordem Coleoptera é a mais diversa em número de espécies, com os mais variados hábitos alimentares. Entre os insetos xilófagos, destacam-se os da Família Cerambycidae e poucos trabalhos relatam a presença de Lamiinae na região amazônica. Desta forma, este trabalho objetivou contribuir para o conhecimento da fauna de Lamiinae da região norte do estado de Mato Grosso. Os insetos foram coletados nos anos de 2008 a 2010, em área florestal, no período noturno, das 18: 00

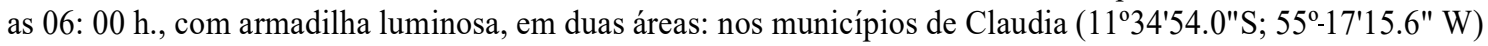
e Sinop ( $\left(1^{\circ} 87^{\prime} 20.9^{\prime \prime} \mathrm{S} 55^{\circ} 47^{\prime} 48.6^{\prime \prime} \mathrm{W}\right)$. No laboratório de Entomologia da Universidade Federal do Mato Grosso (UFMT), Câmpus de Sinop, os insetos foram triados, montados e separados para identificação das espécies. A presente contribuição enumerou 20 espécies de Lamiinae para o estado de Mato Grosso, distribuídos em 11 tribos e 18 gêneros, com quatro novas ocorrências para o estado e oito para a região norte do estado. Grosso e contribui para o conhecimento da riqueza e da distribuição de algumas espécies de Ceramcycidae, os quais constituem bons indicadores de diferentes compartimentos do sistema.
\end{abstract}

Palavras-chave: Amazônia meridional; besouros; Insecta; inventário.

\section{Contribution to the knowledge of LAMIINAE (COLEOPTERA: CERAMBYCIDAE) in the state of Mato Grosso, Brazil}

\begin{abstract}
The Class Insecta, represents $80 \%$ of the Animal Kingdom and order Coleoptera is the most diverse, concerning the number of species, with the most varied eating habits. Among the xylophagous insects stand out from the family Cerambycidae and few studies have reported the presence of Lamiinae in the Amazon region. Thus, this study aimed to contribute to the knowledge of the fauna of Lamiinae from the northern state of Mato Grosso. The insects were collected in the years 2008-2010, in forest area, at night, from 18:00 to 06:00

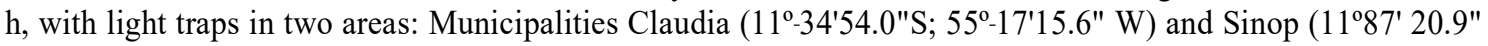
S 55 47' 48.6" W). In entomology laboratory of Universidade Federal de Mato Grosso (UFMT), Campus of Sinop, the insects were sorted, mounted, and separated to identification to species level. The present contribution listed 20 species of Lamiinae for the state of Mato Grosso, distributed in 11 tribes and 18 genera, with four new occurrences for the state and eight for the northern region of the state and contributes to the knowledge of the richness and distribution of some species of Ceramcycidae, which are good indicators of different compartments of the system.
\end{abstract}

Keywords: beetles; Insect; inventory; Southern Amazon.

\section{INTRODUÇÃO}

A ordem Coleoptera representa o agrupamento mais rico em número de espécie de todo o reino Animalia, com mais de 385.000 espécies descritas até o momento (SLIPINSKI et al. 2011), com os mais variados hábitos alimentares. Entre os insetos xilófagos, isto é, os que se alimentam de madeira, destacam-se os da família Cerambycidae, vulgarmente conhecidos como brocas, coleobrocas, serradores e serra-paus (PIRES et al., 2011).

Esses besouros são organismos conspícuos em florestas, onde ocupam desde o solo até a copa das árvores (MONNÉ et al. 2009a). Podem ser polífagos ou monófagos, e apresentam, em vários níveis, uma alta especificidade com sua planta hospedeira (LINSLEY, 1959). Segundo essa mesma autora, as espécies da subfamília Lamiinae e as mais especializadas de Cerambycinae parecem estarem restritas a Angiospermae.
Essas espécies participam ativamente do processo de decomposição da madeira morta e da ciclagem de nutrientes nos ecossistemas florestais (COBB et al., 2010).

Os Lamiinae (Cerambycidae) são a subfamília mais numerosa dentre os Cerambycidae e é extremamente diversa na forma e nos padrões do tegumento (GALILEO; MARTINS, 2006). Caracterizam-se pela cabeça com fronte larga, vertical, ou quase vertical, o último segmento dos palpos fusiforme, quase pontiagudo, o protórax lateralmente arredondado, as tíbias anteriores em sua metade ou terço distal com um sulco oblíquo interno ou posterior e as garras tarsais bífidas, denteadas ou apendiculares.

Com relação à sistemática, Rodrigues et al. (2010) relataram que o grupo é relativamente bem estudado e, no Brasil, conta com aproximadamente 1.000 gêneros com 4.000 espécies (MONNÉ et al., 2009a) porém são escassos os trabalhos sobre a fauna de Cerambycidae em alguns biomas do 
país (MAIA et al., 2003; GALILEO; MARTINS, 2006; MONNÉ et al., 2009b).

Os cerambicídeos estão estritamente associados às plantas hospedeiras e, portanto, são excelentes indicadores do estado de conservação de um ecossistema (MONNÉ et al., 2012). Pode-se registrar a importância de várias famílias como indicadores de qualidade de ambiente (NICHOLS et al., 2008; Gardner et al., 2008) e da degradação ambiental, devido às variadas funções que desempenham na natureza (AZEVEDO et al., 2011). Segundo Rocha et al. (2010), o uso de bioindicadores é essencial para o monitoramento ambiental e as principais características de um bioindicador são: riqueza e diversidade de espécies, fácil manuseio, fidelidade ecológica, fragilidade a pequenas mudanças ambientais e boas (rápidas) respostas do organismo. A Classe Insecta tem todos eles. No entanto, algumas espécies respondem melhor que outras a essas mudanças e de acordo com o meio ambiente.

Alguns trabalhos relatam a presença de Lamiinae na região amazônica, dentre eles podemos destacar: Galileo; Martins (2011); Moysés; Galileo (2009), Monné (2016). Este último autor, cita aproximadamente 317 espécies de Lamiinae para o Mato Grosso, sendo mais de 75 da região norte do Estado. Desta forma, este trabalho pretende contribuir para 0 conhecimento da fauna de Lamiinae da região norte do Estado de Mato Grosso e, assim contribuir para um melhor entendimento da biogeografia das espécies no Brasil.

\section{MATERIAL E MÉTODOS}

Os Lamiinae foram coletados em amostragens bimestrais de 12 horas (das 18:00 as 06:00h) entre os meses de setembro de 2008 a junho de 2010. Foram usadas duas armadilhas luminosas tipo Luiz de Queiroz (lâmpada fluorescente de 20W, luz branca) distanciadas em 50 metros, conforme Barreto et al. (2013) em duas localidades distintas: Fazenda Continental ( $\left.11^{\circ} 34^{\prime} 54.0^{\prime \prime} \mathrm{S} ; 55^{\circ} 17^{\prime} 15.6^{\prime \prime} \mathrm{W}\right)$ no município de

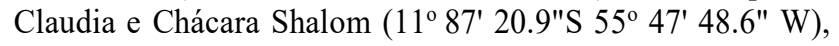
localizada no município de Sinop, MT.

As áreas estudadas correspondem a uma floresta de transição entre Floresta Amazônica e Cerrado. O clima da região é tropical, quente e úmido (Am de Köeppen), com uma estação seca mais prolongada e uma estação úmida de quatro meses, entre dezembro a março. A precipitação pluviométrica média anual é de $2000 \mathrm{~mm}$, muito reduzida no período de maio a agosto. Apresenta uma temperatura média em torno de $24^{\circ} \mathrm{C}$. $\mathrm{O}$ fragmento florestal amostrado é uma área onde foi realizado manejo a 10 anos e sofre forte influência antrópica por ficar próximo a área urbana.

No laboratório de Entomologia da UFMT, Câmpus de Sinop, os insetos foram triados, montados e separados para identificação das espécies. A identificação foi realizada pelo Dr. Miguel A. Monné do Museu Nacional, Universidade Federal do Rio de Janeiro, Rio de Janeiro (MNRJ) e o material estudado encontra-se depositado na coleção entomológica do Acervo Biológico da Amazônia Meridional (ABAM - E) da UFMT.

\section{RESULTADOS}

Do total de 45 exemplares de Lamiinae examinados, quatro são novas ocorrências para o MT e oito para a região norte do estado. Deste total 20 foram identificadas quanto à espécie e cinco exemplares foram identificados somente quanto ao gênero (Hippopsis sp. - 1 ex., Hypsioma sp. - 1 ex., Nealcidion sp. -1 ex., Oreodera spp. 2 ex.).
As espécies amostradas estão distribuídas em 11 tribos, sendo elas Acanthocinini Blanchard, 1845 ( $\mathrm{n}=01)$, Acanthoderini Thomson, $1860(\mathrm{n}=09)$, Acrocinini Thomson, 1860 ( $\mathrm{n}=02)$, Agapanthiini Mulsant, $1839(\mathrm{n}=01)$, Anisocerini Thomson, $1860(\mathrm{n}=04)$, Colobotheini Thomson, $1860(\mathrm{n}=01)$, Desmiphorini Thomson, $1860(\mathrm{n}=01)$, Hemilophini Thomson, $1868(\mathrm{n}=01)$, Lamiini Latreille, 1825 $(\mathrm{n}=01)$, Onciderini Thomson, $1860(\mathrm{n}=03)$ e Polyrhaphidini $(\mathrm{n}=01)$ (Tabela 1$)$.

Registramos como novas ocorrências, na região Meridional, em Mato Grosso as espécies: Desmiphora hirticollis (Olivier, 1795); Colobothea lunulata Lucas, 1857; Onychocerus crassus (Voet, 1778); Oreodera albata Villiers, 1971; Polyrhaphis gracilis Bates, 1862;); Psapharochrus nigricans (Lameere, 1884); Taeniotes farinosus (Linnaeus, 1758) (Tabela 1).

Lamiinae (novos registros para o Mato Grosso):

Oreodera albata Villiers, 1971

Distribuição - Panamá, Venezuela, Guiana Francesa, Peru, Brasil (Amazonas) (Monné, 2018).

Onychocerus crassus (Voet, 1778)

Distribuição - Honduras a América do Sul (amplamente distribuído) e Antilhas, Brasil (Rio de Janeiro (Rodrigues et al., 2010), Maranhão (Martins et al., 2009) e Bahia (Menezes et al., 2012)).

\section{Desmiphora hirticollis (Olivier, 1795)}

Distribuição - Estados Unidos, México, Guatemala, Belize, Honduras, Nicarágua, Costa Rica, Panamá, Colômbia, Venezuela, Guiana Francesa, Bolívia, Argentina, Cuba, Jamaica, São Vicente, Santa Lúcia, Granada, Martinica, Guadalupe, Granadinas, Ilhas Caymã, Curaçau, Porto Rico, Union, Mustique, Ilhas Galápagos. Brasil (Ceará, Maranhão, Bahia, Minas Gerais, Espírito Santo, Rio de Janeiro, São Paulo, Paraná, Santa Catarina, Rio Grande do Sul) (Monné, 2018).

\section{Polyrhaphis gracilis (Bates, 1862)}

Distribuição - Bolívia, Equador, Guiana Francesa e Brasil: Amazonas, São Paulo (Monné, 2018; Santos-Silva et al., 2010).

\section{DISCUSSÃO}

Diversos fatores podem determinam a diversidade das espécies, em condições amplas, como em um fragmento florestal, os mecanismos mais influentes na adição e manutenção da diversidade de organismos associados são a área e a heterogeneidade do hábitat (TRIANTS et al., 2003). Apesar do total de Lamiinae coletados ser considerado pequeno, esse fato pode ser atribuído à metodologia de coleta. Pois, embora a armadilha luminosa seja utilizada para coleta desses insetos (Silveira, 2010; Barreto et al., 2013), as etanólicas (Bernardi et al., 2011) e as tipo Malaise (Holdefer et al., 2014, 2015) também são consideradas atrativas para cerambicídeos em relação à riqueza e abundância de espécies. Mas, também, ao estado fenológico da planta hospedeira (HOLDEFER et al., 2014).

Embora novos registros de ocorrência desses insetos sejam publicados frequentemente (BARRETO et al., 2013; GALILEO; MARTINS, 2010; GALILEO et al., 2014; 
MONNÉ et al., 2012, 2009b; RODRIGUES et al., 2010; SOUZA; SILVA, 2012), dentre outros, neste estudo foram registradas mais oito espécies desconhecidas na região norte do estado. Isto sugere que ainda pouco se conhece sobre a diversidade desses insetos e que se encontra ameaçada pelo elevado grau de degradação ambiental que vem sofrendo.

Makino et al. (2007) relataram que a diversidade de cerambicídeos é elevada em florestas secundárias e que essa variação, quanto à diversidade de espécies, pode ser atribuída às características próprias de cada local, em especial ao tipo de vegetação, sua conservação e quantidade e idade de material em decomposição A abertura do dossel e a variedade de plantas hospedeiras também influenciam a diversidade $\mathrm{O}$ município de Sinop, localizado na região Centro-Norte de Mato Grosso, apresenta desde a década de 1970, um crescimento econômico acelerado quando comparado a outros municípios da região (BRUM et al., 2011). Segundo esses mesmos autores, esse rápido crescimento teve um preço elevado e custou grande parte do capital natural existente, com consequente incremento e intensificação do desmatamento. Esses fatores interferem no ambiente natural e podem comprometer a qualidade da água para a flora e fauna (PRIMO et al., 2011).

Nossos resultados podem contribuir para um melhor entendimento sobre a biogeografia das espécies, pois Menezes et al. (2012) relataram que com o aumento de estudos com Cerambycidae nas diversas regiões brasileiras, conheceremos de forma mais clara e real os padrões de distribuição das suas espécies no Brasil. Alguns desses insetos apresentam considerável importância econômica por possuírem larvas broqueadoras de plantas cultivadas (LAWRENCE et al., 1999). Os Cerambycidae vêm sendo referenciadas pelos bons resultados como bioindicadoras e, ainda, por apresentarem facilidade de coleta e, nas relações com o ecossistema, alta fidelidade ecológica, diversificação taxonômica, e por serem rapidamente afetadas quando ocorre algum tipo de modificação ou fragmentação de ambiente (DIDHAM, 1998; OLIVEIRA et al., 2014).

Tabela 1. Espécies de Lamiinae (Cerambycidae) coletados em duas áreas na região norte de Mato Grosso, Brasil, no período de setembro de 2008 a junho de 2010, com novo registro para Mato Grosso (*).

Table 1. Species of Lamiinae (Cerambycidae) collected in two areas in the northern region of Mato Grosso, Brazil, from September 2008 to June 2010, with new registration for Mato Grosso (*)

\begin{tabular}{|c|c|c|}
\hline Tribo & Espécie & Local e Data Coleta. \\
\hline \multirow[t]{2}{*}{ Acrocinini } & Acrocinus longimanus (Linné, 1758) & BR, Mato Grosso, Claudia. 11.x.2008 \\
\hline & Nealcidion $\mathrm{sp}$. & BR, Mato Grosso, Sinop. 17.ix.2008 \\
\hline \multirow[t]{10}{*}{ Acanthocinini } & Hylettus seniculus (Germar, 1824) & BR, Mato Grosso, Claudia. 11. x.2008 \\
\hline & Myoxomorpha funesta (Erichson, 1847) & BR, Mato Grosso, Sinop. 09.x.2008 \\
\hline & Oreodera aerumnosa Erichson, 1847 & BR, Mato Grosso, Claudia. 09. x.2008 \\
\hline & Oreodera albata Villiers, $1971^{*}$ & BR, Mato Grosso, Sinop. 10. iii.2010 \\
\hline & Oreodera glauca (Linnaeus, 1758) & BR, Mato Grosso, Sinop. 11. x.2008 \\
\hline & Oreodera sp. 1 & BR, Mato Grosso, Sinop. 17. ix.2008 \\
\hline & Oreodera sp. 2 & BR, Mato Grosso, Sinop. 11. x.2008 \\
\hline & Psapharochrus bivittis (Whiote, 1855) & BR, Mato Grosso, Sinop. 02.iv.2010 \\
\hline & Psapharochrus nigricans (Lameere, 1884) & BR, Mato Grosso, Sinop. 26. vii.2009 \\
\hline & Steirastoma breve (Sulzer, 1776) & BR, Mato Grosso, Sinop. 26. vii.2009 \\
\hline \multirow[t]{4}{*}{ Anisocerini } & Onychocerus albitarsis Pescoe, 1859 & BR, Mato Grosso, Sinop.11. x.2008 \\
\hline & Onychocerus concentricus Bates, 1862 & BR, Mato Grosso, Sinop.09. iii.2010 \\
\hline & Onychocerus crassus (Voet, 1778) * & BR, Mato Grosso, Sinop. 11. x.2008 \\
\hline & Platysternus hebraeus (Fabricius, 1781) & BR, Mato Grosso, Sinop. 11. x.2008 \\
\hline Agapanthiini & Hippopsis sp. & BR, Mato Grosso, Sinop. 09. x.2008 \\
\hline Colobotheini & Colobothea lunulata Lucas, 1857 & BR, Mato Grosso, Claudia. 11. x.2008 \\
\hline Desmiphorini & Desmiphora hirticollis (Olivier, 1795) * & BR, Mato Grosso, Claudia. 09. iii.2010 \\
\hline Hemilophini & Adesmus divus (Chabrillac, 1857) & BR, Mato Grosso, Sinop.09. iii.2010 \\
\hline Lamiini & Taeniotes farinosus (Linnaeus, 1758) & BR, Mato Grosso, Sinop.17. ix.2008 \\
\hline \multirow[t]{3}{*}{ Onciderini } & Cipriscola fasciata (Thomson, 1860) & BR, Mato Grosso, Sinop. 15. ix.2008 \\
\hline & Hypsioma sp. & BR, Mato Grosso, Sinop. 26. vii.2009 \\
\hline & Lochmaeocles sladeni (Gahan, 1903) & BR, Mato Grosso, Sinop. 10.iii.2010 \\
\hline Polyrhaphidini & Polyrhaphis gracilis Bates, 1862* & BR, Mato Grosso, Sinop. 24.vii.2009 \\
\hline
\end{tabular}

\section{CONCLUSÕES}

O presente trabalho registra 08 novas ocorrências de Lamiinae para a região meridional na Amazônia Matogrossense, sendo 04 novas ocorrências para o Estado de Mato Grosso e contribui para o conhecimento da riqueza e da distribuição de algumas espécies de Ceramcycidae, os quais constituem bons indicadores de diferentes compartimentos do sistema.

\section{AGRADECIMENTOS}

Ao Dr. Miguel A. Monné, do Museu Nacional da Universidade Federal do Rio de Janeiro, pela identificação dos insetos.

\section{REFERÊNCIAS}

AZEVEDO, F. R.; MOURA, M. A. R. de; ARRAIS, M. S. B.; NERE, D. R. Composição da entomofauna da Floresta Nacional do Araripe em diferentes vegetações e estações do ano. Revista Ceres, Viçosa, v. 58, n. 6, p. 740-748. 2011. DOI: http://dx.doi.org/10.1590/S0034737X2011000600010

BARRETO, M. R.; MACHINER, R.; SMIDERLE, E. C. Cerambycinae (Coleoptera, Cerambycidae) in Mato Grosso, Brazil. Biota Neotropical, Campinas, v.13, n.1, p.331-335. 2013.

BERNARDI, O.; GARCIA, M. S.; ELY E SILVA, E. J.; ZAZYCKI, L. C. F.; BERNARDI, D.; MIORELLI, D.; 
FINKENAUER, E. Besouros cerambycidae associados a Eucalyptus spp. no município de Pinheiro Machado, RS. Ciência Florestal, Santa Maria, v. 21, n. 1, p. 23-30. 2011. DOI: http://dx.doi.org/10.5902/198050982744

BRUM, A. L.; DALFOVO, W. C. T.; BENFICA, V. C. A Relação Entre o Desmatamento e o Crescimento das Culturas de Grãos no Município de Sinop-MT: Uma análise para o período de 1984 a 2009. Desenvolvimento em Questão, Ijuí, v. 9, n. 17, p. 159-190, 2011.

COBB, T. P.; HANNAM, K. D.; KISHCHUK, B. E.; LANGOR, D. W.; QUIDEAU, S. A.; SPENCE, J. R. Wood-feeding beetles and soil nutrient cycling in burned forests: implications of post-fire salvage logging. Agricultural and Forest Entomology, v. 12, n. 1, p. 9-18. 2010. DOI: https://dx.doi.org/10.1111/j.14619563.2009.00440.x

DIDHAM, R.; HAMMOND, P. M.; LAWTON, J. H.; EGGLETON, P.; STORK, N. E. Beetle species responses to tropical forest fragmentation. Ecological Monographs. Lawrence, v. 68, n. 3. p. 295-323. 1998. DOI: https://dx.doi.org/10.1890/00129615(1998)068[0295:BSRTTF]2.0.CO;2

GALILEO, M. H. M.; MARTINS, U. R. Cerambycidae (Coleoptera) - Parque Copesul de Proteção Ambiental, Triunfo, Rio Grande do Sul. Porto Alegre: Museu de Ciências Naturais da Fundação Zoobotânica do Rio Grande do Sul, 2006. 316 p.

GALILEO, M. H. M.; MARTINS, U. R. Novas espécies de Acanthoderini, Onciderini e Pteropliini (Coleoptera: Cerambycidae: Lamiinae) do Brasil e da Bolívia. Acta Amazônica, Manaus, v. 41, n. 1, p. 175-182. 2011. http://dx.doi.org/10.1590/S0044-59672011000100021

GALILEO, M. H. M.; MARTINS, U. R.; FRANCISCO, E. D. L. Cerambycidae (Coleoptera) do Parque Nacional da Serra das Confusões, Piauí, Brasil: novas espécies e novos registros. Iheringia - Série Zoológica, Porto Alegre, v. 103, n. 4, p. 393-397. 2014 http://dx.doi.org/10.1590/S0073-47212013000400010

GALILEO, M. H. M.; MARTINS, U. R. New species of Cerambycinae (Coleoptera, Cerambycidae) from South America. Insecta Mundi, Gainesville, v.115, n.1, p.1-9. 2010.

GARDNER, T. A.; HERNÁNDEZ, M. I. M.; BARLOW, J.; PERES, C. A. Understanding the biodiversity consequences of habitat change: the value of secondary and plantation forests for neotropical dung beetles. Journal Applyed Ecology, v. 45, n. 3, p. 883-893, 2008. https://dx.doi.org/10.1111/j.1365-2664.2008.01454.x

HOLDEFER, D. R.; GARCIA, F. R. M. Análise faunística de cerambicídeos (Coleoptera, Cerambycidae) em floresta subtropical úmida brasileira. Entomotropica, Maracay, v. 30, n. 13, p. 118-134. 2015.

HOLDEFER, D. R.; SARTOR, V.; GARCIA, F. R. M. Flutuação populacional de espécies predominantes de cerambycidae em mata atlântica do sul do Brasil. Interciência, Caracas, v. 39, n. 10, p. 745-750. 2014.

LAWRENCE, F. A.; HASTING, A. M.; DALLWITZ, M. J.; PAINE, T. A.; ZURCHER, E. J. Beetles of the Word: A Key and Information System for Families and Subfamilies. Version 1.0 for MS Windows. Melbourne: CSIRO, 1999.

LINSLEY, E. G., 1959. Ecology of Cerambycidae. Annual Review of Entomology, Stanford, v. 4, n. 1, p. 99-138. https://dx.doi.org/10.1146/annurev.en.04.010159.000531
MAIA, A. C. D.; IANNUZZI, L.; NOBRE, C. E. B.; ALBUQUERQUE, C. M. Padrões locais de diversidade de Cerambycidae (Insecta, Coleoptera) em vegetação de caatinga. In: LEAL, I. R.; TABARELLI, M.; SILVA, J. M. C. (Org.). Ecologia e Conservação da Caatinga. Recife: Editora Universitária da Universidade Federal de Pernambuco, 2003. p. 391-433.

MAKINO, S.; GOTO, H.; HASEGAWA, M.; KIMIKO OKABE, K.; TANAKA, H.; INOUE, T.; OKOCHI, I. Degradation of longicorn beetle (Coleoptera, Cerambucidae, Disteniidae) fauna caused by conversion from broad-leaved to man-made conifer stands of Cryopomeria japonica (Taxodiaceae) in central Japan. Ecological Research, Kyoto, v. 22, n. 3, p. 134-156, 2007. https://dx.doi.org/10.1007/s11284-007-0359-y

MARTINS, U. R.; GALILEO, M. H. M.; LIMEIRA-DEOLIVEIRA, F. Cerambycidae (Coleoptera) do estado do Maranhão, Brasil. Papéis Avulsos de Zoologia, São Paulo, v. 49, n. 19, p. 229-247, 2009. DOI: http://dx.doi.org/10.1590/S0031-10492009003800001

MENEZES, E. C.; SILVA-NETO, A. M.; NASCIMENTO, F. E. L.; BRAVO, F. R. Lista dos Cerambycidae, incluindo 12 Holótipos, Presentes no Museu de Zoologia da Universidade Estadual de Feira de Santana e o Primeiro Registro da espécie Chrysoprasis airi Napp \& Martins para o Brasil. Entomobrasilis, Vassouras, v. 5, n. 1, p. 4958, 2012.

MONNÉ, M. A.; BEZARK, L; HOVORE, F. T. Checklist of the Cerambycidae, or longhorned beetles (Coleoptera) of the Western Hemisphere. BioQuip Publications, v. 1, n.1. p. 1-433, 2009a.

MONNÉ, M. L.; MONNÉ, M. A.; MERMUDES, J. R. M. Inventário das espécies de Cerambycinae (Insecta, Coleoptera, Cerambycidae) do Parque Nacional do Itatiaia, Estado do Rio de Janeiro, Brasil. Biota Neotrópica, Campinas, v. 9, n. 3, p. 283-312. 2009b.

MONNÉ, M. L.; MONNÉ, M. A.; QUINTINO, H. Y.; BOTERO, J. P.; MACHADO, V. S.; ARAGÃO, A. C.; SIMÕES, M. V. P.; CUPELLO, M. Inventory of the Lamiinae species (Insecta, Coleoptera, Cerambycidae) of the Parque Nacional do Itatiaia, RJ, Brazil. Biota Neotropica, Campinas, v. 12, n. 1, p. 39-76, 2012. DOI: http://dx.doi.org/10.1590/S1676-06032012000100004

MONNÉ, M. A. Catalogue of the Cerambycidae (Coleoptera) of the Neotropical Region. Part II. Subfamily Lamiinae. 2016. 1174p.

MONNÉ, M. A. Catalogue of the Cerambycidae (Coleoptera) of the Neotropical Region. Part II. Subfamily Lamiinae. 2018. 1019p.

MOYSÉS, E.; GALILEO, M. H. M. Duas novas espécies de Mauesia (Cerambycidae, Lamiinae, Mauesini) e chave para identificação das espécies do gênero. Papéis Avulsos de Zoologia, São Paulo, v. 49, n. 37, p. 497-501m 2009. DOI:

http://dx.doi.org/10.1590/S003110492009003700001

NICHOLS, E.; LARSEN, T.; SPECTOR, S.; DAVIS, A. L.; ESCOBAR, F.; FAVILA, M.; VULINEC, K. Ecological functions and ecosystem services of Scarabaeine dung beetles. Biologycal Conservation, v.141, n.6, p.14611474. 2008.

DOI: https://dx.doi.org/10.1016/j.biocon.2008.04.011

OLIVEIRA, M. A.; GOMES, C. F. F.; PIRES, E. M.; MARINHO, C. G. S.; DELlA LUCIA, T. M. C. 
Bioindicadores ambientais: insetos como um instrumento desta avaliação. Revista Ceres, Viçosa, v. 61, p. 800-807, 2014. DOI: http://dx.doi.org/10.1590/0034737x201461000005

PIRES, E. M.; MOREIRA, I.; SOARES, M. A.; MARINHO, J. A.; PINTO, A.; ZANUNCIO, J. C. Oxymerus aculeatus (Coleoptera: Cerambycidae) causing damage on corn plants (Zea mays) in Brazil. Revista Colombiana de Entomolologia, v. 37, n. 1, p. 82-83, 2011.

PRIMO, D. C.; MENEZES, R. S. C.; SILVA, T. O. Substâncias húmicas da matéria orgânica do solo: uma revisão de técnicas analíticas e estudos no nordeste brasileiro. Scientia Plena, v. 7, n. 5, p. 1-13, 2011.

ROCHA, J. R. M.; ALMEIDA, J. R.; LINS, G. A., DURVAL, A. Insects as Indicators of Environmental Changing and Pollution: A Review of Appropriate Species and their Monitoring. HOLOS Environment, Rio Claro, v. 10, n. 2, p. 250-262, 2010. DOI: http://dx.doi.org/10.14295/holos.v10i2.2996

RODRIGUES, J. M. S.; MONNÉ, M. A.; MERMUDES, J. R. M. Inventory of the Cerambycidae species (Coleoptera) from Vila Dois Rios (Ilha Grande, Angra dos Reis, Rio de Janeiro, Brazil). Biota Neotropica, Campinas, v. 10, n. 3, p. 311-321. 2010. DOI: http://dx.doi.org/10.1590/S167606032010000300029
SANTOS-SILVA, A.; MARTINS, U. R.; TAVAKILIAN, G. L. Revisão do gênero Polyrhaphis Audinet-Serville (Coleoptera, Cerambycidae, Lamiinae). Papéis Avulsos de Zoologia, São Paulo, v. 50, n. 30, p. 451-509. 2010. DOI: http://dx.doi.org/10.1590/S003110492010003000001

SILVEIRA, M. A. P. A. Análise faunística de insetos nas margens do alto rio Madeira. 2010. 74f. Tese (Doutorado em Agronomia) - Escola Superior de Agronomia Luiz de Queiroz, Porto Velho, 2010.

SLIPINSKI, S. A.; LESCHEN, R. A. B.; LAWRENCE, J. F. Order Coleoptera Linnaeus. In: ZHANG, Z. Q. (Ed.). Animal biodiversity: an outline of higher-level classification and survey of taxonomic richness. Zootaxa, v.3148, n.1, p.1-237, 2011. p. 203-208.

SOUZA, D. D. S.; SILVA, A. D. A. Cerambycidae (Insecta: Coleoptera) of the Parque Natural Municipal de Porto Velho, Rondônia, Western Amazon, Brazil. Biota Neotropica, Campinas, v. 12, n. 1, p. 237-240, 2012. http://dx.doi.org/10.1590/S1676-06032012000100018

TRIANTS, K. A.; MYLONAS, M.; LIKA, K.; VARDINOYANNIS, K. A model for the species - area habitat relationship. Journal of Biogeography, Oxford, v. 30 , n. 1, p. 19-27, 2003. DOI: https://dx.doi.org/10.1046/j.1365-2699.2003.00805.x 